\title{
STRESS CONCENTRATION AT THE CIRCULAR HOLE OF CYCLICALLY BENT LAYERED COMPOSITE PLATE WITHIN THE FRAMEWORK OF A MOMENT THEORY OF THERMOVISCOELASTICITY
}

\author{
Andrzej Katunin \\ Institute of Fundamentals of Machinery Design, Silesian University of Technology \\ Gliwice, Poland \\ andrzej.katunin@polsl.pl
}

\begin{abstract}
In the continuum mechanics there is a class of problems that cannot be solved directly or the solutions of these problems are affected by large errors when the classical equations of thermoviscoelasticity are considered. The paper discusses a special case of such problems - the cyclic bending of a composite plate with a circular hole subjected to the stationary self-heating, which was solved within the framework of a moment theory of thermoviscoelasticity.
\end{abstract}

Keywords: moment theory of thermoviscoelasticity, self-heating effect, composite plate

\section{Introduction}

The classical theory of viscoelasticity cannot explain several phenomena occurring in the mechanical structures, e.g. the behaviour of polycrystalline bodies under the complex loading, and cannot describe satisfactorily a number of other problems, e.g. the stress concentrations near holes, cutouts and cracks in structural elements and others $[1,2]$. The difficulties occur due to the singularities of the particular solutions of these problems. In some of them, due to discontinuities and singularities, the obtained solutions are quite far from the experimental results. However, considering the disagreements between theoretical models and experimental results other approach should be applied to solve the mentioned class of problems, which considers the full stress state including the principal moments of forces acting on the element of the body.

The first systematic studies on the moment (or asymmetric) theory of elasticity were introduced by Cosserat and Cosserat [3], which considered not only the classical stresses, but also the moment stresses occurred during complex loading states or specific geometry of the considered medium. The proposed theory was not applied and developed for over 50 years, until further studies in the area of continuum mechanics were performed. 
The intensive studies concerned with the moment theory of viscoelasticity and thermoviscoelasticity were introduced in the late 60 s of 20 th century, mainly by Russian and Ukrainian researchers [1, 4-6]. The moment theory of viscoelasticity and thermoviscoelasticity was then developed by several researchers $[7,8]$ and is developed until now, mainly by Pobedrya and his followers [9-11]. One of the fundamental studies on the moment theory of thermoviscoelasticity in layered composites is a paper of Pobedrya and Rodriguez [9], which presented the coupled thermomechanical governing equations for behaviour of such structures under non-stationary temperature field. Further studies [10-14] presented a method of the determination of material functions for elastic and viscoelastic media, which is one of the main problems in the considered theory. The application of moment theory for viscoelastic plates was also developed and discussed by Altenbach and Eremeyev in $[15,16]$.

The previous studies, concerned with the self-heating effect in polymeric composites $[17,18]$, show that there are two states of self-heating temperature evolution: the stationary state (when the temperature grows rapidly and stabilizes on the certain value) and the non-stationary state (when the temperature grows following the three-phase model until the breakdown of the structure). In this paper, the solution of particular problem of quasi-static self-heating of cyclically bent plate with a circular hole within the framework of moment theory of thermoviscoelasticity was investigated. The construction of descriptive equations for the investigated problem is based on the analogy between Hooke's law and a theory of linear viscoelasticity. As a result of this study, the equation of moments over the contour of a hole was presented. The applied viscoelastic analogy to the elastic problem with taking into consideration "moment" stresses could be successfully used for solution of time- and temperature-dependent viscoelastic problems.

\section{Basic relations and assumptions}

The stress-strain relation for the linear thermoviscoelastic anisotropic body in the case of stress relaxation has a form:

$$
\sigma_{i j}=\int_{0}^{t} R_{i j k l}\left(t-t^{\prime}, T\right) \frac{\mathrm{d} \varepsilon_{k l}\left(t^{\prime}\right)}{\mathrm{d} t^{\prime}} \mathrm{d} t^{\prime},
$$

where $\boldsymbol{\sigma}$ and $\boldsymbol{\varepsilon}$ are the stress and strain tensors, respectively, and $\mathbf{R}\left(t-t^{\prime}, T\right)$ is a tensor of time- and temperature-dependent relaxation kernels.

Considering the analogy between equations of linear elastic and linear viscoelastic bodies and applying the Laplace transform [16]

$$
\bar{f}(s)=\int_{0}^{\infty} f(t) e^{-s t} \mathrm{~d} t,
$$


to (1) one obtains the Hooke-like equation:

$$
\overline{\boldsymbol{\sigma}}=\overline{\mathbf{R}}(s) \overline{\boldsymbol{\varepsilon}} .
$$

Following this and considering the layered structure of a composite, governing equations of the moment theory of linear thermoviscoelasticity can be presented in the similar form (cf. [11]):

$$
\begin{aligned}
& \bar{\sigma}_{i j}^{(p)}=\bar{S}_{i j k l}^{(p)} u_{k, l}^{(p)}+\bar{s}_{i j k l}^{(p)} \boldsymbol{\kappa}_{k l}^{(p)}, \\
& \bar{\mu}_{i j}^{(p)}=\bar{Q}_{i j k l}^{(p)} \boldsymbol{u}_{k, l}^{(p)}+\bar{q}_{i j k l}^{(p)} \boldsymbol{\kappa}_{k l}^{(p)},
\end{aligned}
$$

where $\boldsymbol{\mu}$ is the moment stress tensor, $\mathbf{S}, \mathbf{s}, \mathbf{Q}, \mathbf{q}$ are the asymmetric tensors of time- and temperature-dependent stress relaxation kernels. All above quantities are referred to the $p$-th layer of a composite, where $p=1,2, \ldots, P$.

In this study the small Cauchy strain and small rotations are assumed:

$$
\bar{\varepsilon}_{i j}=\frac{1}{2}\left(u_{i, j}+u_{j, i}\right), \kappa_{i j}=\omega_{j, i}, \omega_{i}=\frac{1}{2} \delta_{i j k} u_{k, j},
$$

where $\delta_{i j k}$ is the permutation tensor.

For the plane problem of a layered composite plate the displacements have the following form:

$$
u=u_{0}-z \frac{\partial w_{0}}{\partial x}, v=v_{0}-z \frac{\partial w_{0}}{\partial y},
$$

where $-h / 2 \leq z \leq h / 2$ is a thickness coordinate and $h$ is a thickness of a plate.

Using [19] and the above-discussed transformation the stress-strain relations for the plane problem are:

$$
\begin{gathered}
\frac{1}{2}\left(\bar{\sigma}_{i j}^{(p)}+\bar{\sigma}_{j i}^{(p)}\right)=\frac{\bar{E}^{(p)} z_{p}}{1+\bar{v}^{(p)}} \bar{\varepsilon}_{i j}+\frac{\bar{v}^{(p)} \bar{E}^{(p)} z_{p}}{\left(1+\bar{v}^{(p)}\right)\left(1-2 \bar{\nu}^{(p)}\right)} \bar{\varepsilon}_{k k} \delta_{i j}, \\
\bar{\mu}_{i j}^{(p)}=\frac{1}{3} \bar{\mu}_{k k}^{(p)} \delta_{i j}=4 \kappa_{i j}^{(p)} z_{p}\left(\bar{B}_{1}^{(p)}+\bar{B}_{2}^{(p)}\right),
\end{gathered}
$$

where

$$
\bar{E}^{(p)}=\int_{0}^{t} E^{(p)}\left(t-t^{\prime}, T\right) \mathrm{d} t^{\prime}, \bar{v}^{(p)}=\int_{0}^{t} v^{(p)}\left(t^{\prime}\right) \mathrm{d} t^{\prime}, \bar{B}_{i}^{(p)}=\int_{0}^{t} B_{i}^{(p)}\left(t-t^{\prime}, T\right) d t^{\prime},
$$

Here $\bar{B}_{i}$ are the "moment" relaxation kernels. Following the fact that the linear thermoviscoelastic model is considered the time-temperature superposition principle 
can be applied for (8), when the stationary self-heating is analyzed (i.e. the quasi-static problem of thermoviscoelasticity is considered):

$$
\mathrm{d} \tilde{t}=\frac{1}{a_{T}(t)} \mathrm{d} t, \tilde{t}=\int_{0}^{t} \frac{1}{a_{T}(\xi)} \mathrm{d} \xi, \mathrm{d} \tilde{\tau}=\frac{1}{a_{T}(\tau)} \mathrm{d} \tau, \tau=\int_{0}^{\tau} \frac{1}{a_{T}(\xi)} \mathrm{d} \xi,
$$

where $\sim$ denote reduced times with respect to real times and $a_{T}$ is a horizontal shift factor, which could be determined from the Arrhenius relationship [19].

\section{Bending of the plate}

Considering the moment stresses and the above-presented assumptions, the plain problem of bending of a thin viscoelastic plate can be described using the following set of equations:

$$
\begin{gathered}
\bar{\sigma}_{x x}^{(p)}=\frac{\bar{E}^{(p)} z_{p}}{1-\left(\bar{v}^{(p)}\right)^{2}}\left(\frac{\partial^{2} w}{\partial x^{2}}+\bar{v}^{(p)} \frac{\partial^{2} w}{\partial y^{2}}\right), \\
\bar{\sigma}_{y y}^{(p)}=-\frac{\bar{E}^{(p)} z_{p}}{1-\left(\bar{v}^{(p)}\right)^{2}}\left(\frac{\partial^{2} w}{\partial y^{2}}+\bar{v}^{(p)} \frac{\partial^{2} w}{\partial x^{2}}\right), \\
\bar{\tau}_{x y}^{(p)}=\bar{\tau}_{y x}^{(p)}=\frac{\bar{E}^{(p)} z_{p}}{1+\bar{v}^{(p)}} \frac{\partial^{2} w}{\partial x \partial y} \\
\bar{\mu}_{x x}^{(p)}=4 z_{p}\left(\bar{B}_{1}^{(p)}+\bar{B}_{2}^{(p)}\right) \frac{\partial^{2} w}{\partial x \partial y} \\
\bar{\mu}_{y y}^{(p)}=-4 z_{p}\left(\bar{B}_{1}^{(p)}+\bar{B}_{2}^{(p)}\right) \frac{\partial^{2} w}{\partial x \partial y} \\
\bar{\mu}_{x y}^{(p)}=-\bar{\mu}_{y x}^{(p)}=-4 z_{p}\left(\bar{B}_{1}^{(p)} \frac{\partial^{2} w}{\partial x^{2}}+\bar{B}_{2}^{(p)} \frac{\partial^{2} w}{\partial y^{2}}\right),
\end{gathered}
$$

where $x, y$ are the Cartesian coordinates, $\tau_{i j}$ are the shear components of a stress tensor and $w=w(x, y)$.

Following the statement that the plane problem is considered some elements of the moment stress tensor could be omitted:

$$
\bar{\mu}_{z z}=\bar{\mu}_{x z}=\bar{\mu}_{y z}=\bar{\mu}_{z x}=\bar{\mu}_{z y}=0 .
$$


Considering (10) and the decomposition of the shear stress components into axiator and deviator parts:

$$
\bar{\tau}_{i j}^{(p)}=\bar{\tau}_{i j}^{(p)(A)}+\bar{\tau}_{i j}^{(p)(D)}, \bar{\tau}_{i j}^{(p)(A)}=\frac{1}{2}\left(\bar{\tau}_{i j}^{(p)}-\bar{\tau}_{j i}^{(p)}\right), \bar{\tau}_{i j}^{(p)(D)}=\frac{1}{2}\left(\bar{\tau}_{i j}^{(p)}+\bar{\tau}_{j i}^{(p)}\right),
$$

one can write the equilibrium equations for the bent plate (cf. [20]):

$$
\begin{gathered}
\frac{\partial \bar{\sigma}_{x x}^{(p)}}{\partial x}+\frac{\partial \bar{\tau}_{y x}^{(p)(D)}}{\partial y}+\frac{\partial \bar{\tau}_{z x}^{(p)(D)}}{\partial z}+\frac{\partial \bar{\tau}_{z x}^{(p)(A)}}{\partial z}=0, \\
\frac{\partial \bar{\tau}_{x y}^{(p)(D)}}{\partial x}+\frac{\partial \bar{\sigma}_{y y}^{(p)}}{\partial y}+\frac{\partial \bar{\tau}_{z y}^{(p)(D)}}{\partial z}-\frac{\partial \bar{\tau}_{y z}^{(p)(A)}}{\partial z}=0, \\
\frac{\partial \bar{\tau}_{x z}^{(p)(D)}}{\partial x}+\frac{\partial \bar{\tau}_{y z}^{(p)(D)}}{\partial y}+\frac{\partial \bar{\sigma}_{z z}^{(p)}}{\partial z}-\frac{\partial \bar{\tau}_{z x}^{(p)(A)}}{\partial x}-\frac{\partial \bar{\tau}_{y z}^{(p)(A)}}{\partial y}=0, \\
\frac{\partial \bar{\mu}_{x x}^{(p)}}{\partial x}+\frac{\partial \bar{\mu}_{y x}^{(p)}}{\partial y}+2 \bar{\tau}_{y z}^{(p)(A)}=0, \\
\frac{\partial \bar{\mu}_{y x}^{(p)}}{\partial x}+\frac{\partial \bar{\mu}_{y y}^{(p)}}{\partial y}+2 \bar{\tau}_{z x}^{(p)(A)}=0 .
\end{gathered}
$$

For the fundamental equation of bending of thin plates

$$
\nabla^{2} \nabla^{2} w=\frac{q}{\bar{D}+4 \bar{B}_{1} h}
$$

where $q=\bar{\sigma}_{z z}^{(p)}$, the resultant bending $M_{i}$ and shear $H_{i}$ moments and the shear forces $N_{i}$ acting in the cross-section of the layered plate, which can be determined by the integration of stresses over the plate thickness, can be presented as follows:

$$
\begin{aligned}
& M_{x}=\sum_{p=1}^{P} \int_{z_{p-1}}^{z_{p}}\left(\bar{\sigma}_{x x}^{(p)} z_{p}+\bar{\mu}_{x y}^{(p)}\right) \mathrm{d} z, \\
& M_{y}=\sum_{p=1}^{P} \int_{z_{p-1}}^{z_{p}}\left(\bar{\sigma}_{y y}^{(p)} z_{p}-\bar{\mu}_{y x}^{(p)}\right) \mathrm{d} z, \\
& H_{x}=\sum_{p=1}^{P} \int_{z_{p-1}}^{z_{p}}\left(\bar{\tau}_{x y}^{(p)} z_{p}-\bar{\mu}_{x x}^{(p)}\right) \mathrm{d} z,
\end{aligned}
$$




$$
\begin{gathered}
H_{y}=\sum_{p=1}^{P} \int_{z_{p-1}}^{z_{p}}\left(\bar{\tau}_{y x}^{(p)} z_{p}+\bar{\mu}_{y y}^{(p)}\right) \mathrm{d} z, \\
N_{x}=\sum_{p=1}^{P} \int_{z_{p-1}}^{z_{p}}\left(\bar{\tau}_{x z}^{(p)(D)}-\bar{\tau}_{z x}^{(p)(A)}\right) \mathrm{d} z, \\
N_{y}=\sum_{p=1}^{P} \int_{z_{p-1}}^{z_{p}}\left(\bar{\tau}_{y z}^{(p)(D)}+\bar{\tau}_{y z}^{(p)(A)}\right) \mathrm{d} z .
\end{gathered}
$$

Equations (15) could be transformed into the following form:

$$
\begin{gathered}
M_{x}=-\left(\bar{a} \frac{\partial^{2} w}{\partial x^{2}}+\bar{b} \frac{\partial^{2} w}{\partial y^{2}}\right), M_{y}=-\left(\bar{a} \frac{\partial^{2} w}{\partial y^{2}}+\bar{b} \frac{\partial^{2} w}{\partial x^{2}}\right), \\
H_{x}=H_{y}=(\bar{b}-\bar{a}) \frac{\partial^{2} w}{\partial x \partial y}, \\
N_{x}=-\bar{a} \frac{\partial}{\partial x}\left(\frac{\partial^{2} w}{\partial x^{2}}+\frac{\partial^{2} w}{\partial y^{2}}\right), N_{y}=-\bar{a} \frac{\partial}{\partial y}\left(\frac{\partial^{2} w}{\partial x^{2}}+\frac{\partial^{2} w}{\partial y^{2}}\right),
\end{gathered}
$$

where

$$
\bar{a}=\bar{D}+4 \bar{B}_{1} h, \bar{b}=\bar{v} \bar{D}-4 \bar{B}_{2} h .
$$

Considering the moments, the final form of stresses could be presented as:

$$
\begin{gathered}
\bar{\sigma}_{x x}^{(p)}=-\frac{\bar{E}^{(p)} z_{p}}{1-\left(\bar{v}^{(p)}\right)^{2}}\left(\frac{\bar{a} M_{x}-\bar{b} M_{y}}{\bar{b}^{2}-\bar{a}^{2}}+\bar{v}^{(p)} \frac{\bar{a} M_{y}-\bar{b} M_{x}}{\bar{b}^{2}-\bar{a}^{2}}\right), \\
\bar{\sigma}_{y y}^{(p)}=-\frac{\bar{E}^{(p)} z_{p}}{1-\left(\bar{v}^{(p)}\right)^{2}}\left(\frac{\bar{a} M_{y}-\bar{b} M_{x}}{\bar{b}^{2}-\bar{a}^{2}}+\bar{v}^{(p)} \frac{\bar{a} M_{x}-\bar{b} M_{y}}{\bar{b}^{2}-\bar{a}^{2}}\right), \\
\bar{\tau}_{x y}^{(p)}=\bar{\tau}_{y x}^{(p)}=-\frac{\bar{E}^{(p)} z_{p}}{1+\left(\bar{v}^{(p)}\right)^{2}} \frac{H}{\bar{b}-\bar{a}}, \\
\bar{\tau}_{x z}^{(p)}=-\frac{N_{x}}{\bar{a}}\left(\frac{4 z_{p}^{2}-h^{2}}{8\left(1-\left(\bar{v}^{(p)}\right)^{2}\right)}-4 \bar{B}_{1}^{(p)}\right),
\end{gathered}
$$




$$
\begin{gathered}
\bar{\tau}_{y z}^{(p)}=-\frac{N_{y}}{\bar{a}}\left(\bar{E}^{(p)} \frac{4 z_{p}^{2}-h^{2}}{8\left(1-\left(\bar{v}^{(p)}\right)^{2}\right)}-4 \bar{B}_{1}^{(p)}\right), \\
\bar{\mu}_{x x}^{(p)}=-\bar{\mu}_{y y}^{(p)}=4 H\left(\frac{\bar{B}_{1}^{(p)}+\bar{B}_{2}^{(p)}}{\bar{b}-\bar{a}}\right), \\
\bar{\mu}_{x y}^{(p)}=-4\left(\bar{B}_{1}^{(p)} \frac{\bar{a} M_{x}-\bar{b} M_{y}}{\bar{b}^{2}-\bar{a}^{2}}-\bar{B}_{2}^{(p)} \frac{\bar{a} M_{y}-\bar{b} M_{x}}{\bar{b}^{2}-\bar{a}^{2}}\right), \\
\bar{\mu}_{y z}^{(p)}=4\left(\bar{B}_{1}^{(p)} \frac{\bar{a} M_{y}-\bar{b} M_{x}}{\bar{b}^{2}-\bar{a}^{2}}+\bar{B}_{2}^{(p)} \frac{\bar{a} M_{x}-\bar{b} M_{y}}{\bar{b}^{2}-\bar{a}^{2}}\right) .
\end{gathered}
$$

Assuming pure bending of a plate

$$
M_{x}^{0}=M, M_{y}^{0}=H^{0}=N_{x}^{0}=N_{y}^{0}=0
$$

the displacements of the bent plate take a form:

$$
w_{0}=\frac{M\left(\bar{a} x^{2}-\bar{b} y^{2}\right)}{2\left(\bar{b}^{2}-\bar{a}^{2}\right)} .
$$

Considering the boundary conditions based on [20]:

$$
\varphi_{0}=\frac{M z_{p}}{4(\bar{b}+\bar{a})}, \psi_{0}=\frac{M z_{p}}{2(\bar{b}-\bar{a})},
$$

one obtains the equation for the moments over the contour of a circular hole:

$$
M_{\theta}=M\left(1-2 \cos 2 \theta\left(\frac{\left(h / \bar{\vartheta}^{(p)}\right)^{2}\left(1+\bar{v}^{(p)}\right)+24\left(1-\bar{v}^{(p)}\right)\left(1+\bar{\eta}^{(p)}\right)}{\left(h / \bar{\vartheta}^{(p)}\right)^{2}\left(3+\bar{v}^{(p)}\right)+24\left(1-\bar{v}^{(p)}\right)\left(3+\bar{\eta}^{(p)}\right)}\right)\right),
$$

where

$$
\bar{\vartheta}^{(p)}=\left(\frac{2 \bar{B}_{1}^{(p)}\left(1+\bar{v}^{(p)}\right)}{\bar{E}^{(p)}}\right)^{\frac{1}{2}}, \bar{\eta}^{(p)}=\frac{\bar{B}_{2}^{(p)}}{\bar{B}_{1}^{(p)}} .
$$

The material functions $\bar{B}_{i}$ cannot be determined experimentally, which represents the main problem of applicability of the considered theory in practical problems. 
However, following the studies presented in $[11,13,14]$ this problem could be particularly solved by the application of a homogenization method used in the firstorder approximation theory. In [14] Omarov presented the example of a determination of "moment" material functions for the elastic medium. Following the presented approach it is possible to obtain the "moment" relaxation kernels for the thermoviscoelastic medium in the same manner.

Considering results for the elastic problem presented in [21], the deflections of a plate with a circular hole within the classical hypotheses are overestimated with respect to the moment theory due to taking into account the moment stresses in the latter. The same relationship is applicable for the considered problem of linear thermoviscoelasticity of cyclically bent plate with a circular following the applied viscoelastic analogy to the elastic formulation of a problem.

\section{Conclusions}

In the following study the theoretical formulation of linear thermoviscoelasticity with taking into account moment stresses were presented for a specific problem of cyclic bending of a composite layered plate with a circular hole. Following this approach, the governing equations of linear theory of thermoviscoelasticity were formulated for a stationary state based on the solution of similar elastic problem and application of viscoelastic analogy and time-temperature superposition principle. Such formulation could be useful for modelling of similar problems in order to determine the true stress concentrations and bending moment in plates with holes subjected to cyclic loading.

Due to the intensively developed generalizations of formulations of isotropic elastic media with consideration of moment stresses to orthotropic [21-23] and anisotropic $[2,24]$ ones, the approach of viscoelastic analogy and time-temperature superposition could be used for obtaining similar formulations for linear visco- and thermoviscoelasticity as well.

\section{Acknowledgements}

The research was carried out within the framework of Mentoring FNP programme supported by the Foundation for Polish Science. The author thanks Prof. Pedro P. Camanho from the University of Porto for comments and recommendations during the preparation of this paper.

\section{References}

[1] Savin G.N., Nemish Y.N., Investigations into stress concentration in the moment theory of elasticity (a survey), Appl. Mech. 1968, 4, 1-17.

[2] Altenbach J., Altenbach H., Eremeyev V.A., On generalized Cosserat-type theories of plates and shells: a short review and bibliography, Arch. Appl. Mech. 2010, 80, 73-92. 
[3] Cosserat E.F., Cosserat F.F., Théorie des corps deformables, A. Hermann et Fils, Paris 1909.

[4] Kubenko V.D., Shul'ga N.A., A dynamic plane problem of the moment theory of elasticity and viscoelasticity, Appl. Mech. 1967, 3, 50-53.

[5] Lomakin V.A., Savova L.N., Problems of straining of microinhomogeneous viscoelastic bodies and the moment theory of viscoelasticity, Polym. Mech. 1967, 2, 213-220.

[6] Savova L.N., Two-dimensional problem in the moment theory of viscoelasticity concerning stress concentration near a circular hole, Appl. Mech. 1968, 4, 6-13.

[7] Bykov D.L., Il'yushin A.A., Ogibalov P.M., Pobedrya B.E., Some fundamental problems of the theory of thermoviscoelasticity, Polym. Mech. 1971, 1, 41-58.

[8] Kolokol'chikov V.V., Microrelaxation model for describing the moment effects in viscoelastic polymers, Polym. Mech. 1977, 13, 501-506.

[9] Pobedrya B.E., Rodriguez A., Modelling of structures in the mechanics of composites, Mech. Compos. Mater. 2001, 37, 459-474.

[10] Omarov S.E., Effective characteristics of layered composites with allowance for structural changes, Mech. Solids 2007, 42, 463-470.

[11] Pobedrya B.E., Omarov S.E., Determination of material functions for the linear moment theory of viscoelasticity, Mosc. Univ. Mech. Bull. 2007, 62, 117-122.

[12] Pobedrya B.E., Omarov S.E., Constitutive relations of the moment theory of elasticity, Mosc. Univ. Mech. Bull. 2007, 62, 84-86.

[13] Omarov S.E., Determining the material constant in the equilibrium problem for an infinite elastic plane weakened by a circular hole, Mech. Solids 2007, 44, 776-780.

[14] Omarov S.E., A method of determining the material functions in the linear moment theory of elasticity, Mosc. Univ. Mech. Bull. 2009, 64, 110-113.

[15] Altenbach H., Eine direkt formulierte lineare Theorie für viskoelastische Platten und Schalen, Ingeneur Archiv 1988, 58, 215-228.

[16] Altenbach H., Eremeyev V.A., On the bending of viscoelastic plates made of polymer foams, Acta Mech. 2009, 204, 137-154.

[17] Katunin A., Fidali M., Self-heating of polymeric laminated composite plates under the resonant vibrations: theoretical and experimental study, Polym. Compos. 2012, 33, 138-146.

[18] Katunin A., Fidali M., Fatigue and thermal failure of polymeric composites subjected to cyclic loading, Adv. Compos. Lett. 2012, 21, 64-69.

[19] Katunin A., Gnatowski A., Influence of heating rate on evolution of dynamic properties of polymeric laminates, Plast. Rubber Compos. 2012, 41, 233-239.

[20] Gevorkyan G.A., Equations of plate bending in the moment theory of elasticity, Appl. Mech. 1966, 2, 74-79.

[21] Bouyge F., Jasiuk I., Boccara S., Ostoja-Staszewski M., A micromechanically based couplestress model of an elastic orthotropic two-phase composite, Eur. J. Mech. A-Solids 2002, 21, 465-481.

[22] Hayrapetyan G.S., Sargsyan S.H., Theory of micropolar orthotropic elastic thin plates, Comm. Acad. Sci., Armenia 2012, 65, 22-33 (in Russian).

[23] Tsiatas G.C., Yiotis A.J., A modified couple stress theory for bending, buckling and vibration of skew orthotropic micro-plates, Proc. 10 $0^{\text {th }}$ HSTAM Int. Congress of Mechanics, Chania 2013.

[24] Trovalusci P., Masiani R., Non-linear micropolar and classical continua for anisotropic discontinuous materials, Int. J. Solids Struct. 2003, 40, 1281-1297. 
